\title{
NONLINEAR MAPS PRESERVING CONDITION SPECTRUM OF JORDAN SKEW TRIPLE PRODUCT OF OPERATORS
}

\author{
H. Benbouziane, Y. Bouramdane, M. Ech-Cherif El Kettani \\ AND A. LAHSSAINI
}

Abstract. Let $\mathscr{B}(\mathscr{H})$ the algebra of all bounded linear operators on a complex Hilbert space $\mathscr{H}$ with $\operatorname{dim} \mathscr{H} \geqslant 3$. Let $\mathscr{W}, \mathscr{V}$ be subsets of $\mathscr{B}(\mathscr{H})$ which contain all rank-one operators. Denote by $r_{\varepsilon}(A)$ the condition spectral radius of $A \in \mathscr{B}(\mathscr{H})$. We determine the form of surjective maps $\phi: \mathscr{W} \rightarrow \mathscr{V}$ satisfying $r_{\varepsilon}\left(A B^{*} A\right)=r_{\varepsilon}\left(\phi(A) \phi(B)^{*} \phi(A)\right)$ for all $A, B$ in $\mathscr{W}$, we characterize also the structure of surjective maps $\phi: \mathscr{B}(\mathscr{H}) \rightarrow \mathscr{B}(\mathscr{H})$ with $\sigma_{\varepsilon}\left(A B^{*} A\right)=$ $\sigma_{\varepsilon}\left(\phi(A) \phi(B)^{*} \phi(A)\right)$ for all $A, B$ in $\mathscr{B}(\mathscr{H})$ where $\sigma_{\varepsilon}(A)$ is the $\varepsilon$-condition spectrum of an operator $A$ in $\mathscr{B}(\mathscr{H})$.

Mathematics subject classification (2010): Primary 47B49, Secondary 47B48, 47A10, 46H05.

Keywords and phrases: Condition spectra, condition spectral radius, nonlinear Jordan skew triple product preservers.

\section{REFERENCES}

[1] H. Benbouziane, Y. Bouramdane, M. Ech-Cherif El Kettani, A. Lahssaini, Nonlinear commutant preservers, Linear and Multilinear Algebra, (2017), doi:10.1080/03081087.2017. 1307915.

[2] M. Bendaoud, A. Benyouness, M. Sarih, Condition spectra of special operators and condition spectra preservers, J. Math. Anal. Appl. (2017), http://dx.doi.org/10.1016/j.jmaa. 2016.12.022.

[3] M. Bendaoud, A. Benyouness, M. SARIH, Preservers of pseudo spectral radius of operator products, Linear Algebra Appl. 489 (2016) 186-198.

[4] R. Bhatia, P. ŠEMrL, A. R. Sourour, Maps on matrices that preserve the spectral radius distance, Studia Math. 134 (1999) 99-110.

[5] A. Bourhim, T. Jari, J. Mashreghi, Peripheral local spectrum preservers and maps increasing the local spectral radius, Oper. Matrices 1 (2016) 189-208.

[6] J. Cui, V. Forstall, C. K. Li, V. Yannello, Properties and preservers of the pseudospectrum, Linear Algebra Appl. 436 (2012) 316-325.

[7] J. CuI, C. K. LI, Y. T. Poon, Pseudospectra of special operators and pseudospectrum preservers, J. Math. Anal. Appl. 419 (2014) 1261-1273.

[8] J. CUI, C. K. Li, AND N. S. SZE, Unitary similarity invariant function preservers of skew products of operators, J. Math. Anal. Appl. (2017), http://dx.doi.org/10.1016/j . jmaa.2017.04.072.

[9] M. ECH-CHÉRIF El KeTtANI AND H. BENBOUZIANE, Additive maps preserving operators of inner local spectral radius zero, Rendiconti del Circolo Matematico di Palermo 63 (2) (2014) 311-316.

[10] J. HoU, K. HE, X. ZHANG, Nonlinear maps preserving numerical radius of indefinite skew products of operators, Linear Algebra Appl. 430 (2009) 2240-2253.

[11] A. A. JAFARIAN, A. R. Sourour, Linear maps that preserve the commutant, double commutant or the lattice of invariant subspaces, Linear and Multilinear Algebra. 38 (1994) 117-129.

[12] A. Krishnan And S. H. KulKarni, Pseudo spectrum of element in Banach algebra, Oper. Matrices 1 (2017) 263-287.

[13] S. H. Kulkarni, D. Sukumar, The condition spectrum, Acta Sci. Math. (Szeged) 74 (2008) 625641. 
934 H. Benbouziane, Y. Bouramdane, M. Ech-Cherif El Kettani and A. LahsS Aini

[14] G. K. KUMAR, S. H. KULKARNI, Linear maps preserving pseudospectrum and condition spectrum, Banach J. Math. Anal. 6 (2012) 45-60.

[15] C. K. LI, P. ŠEMRL, N. K. Tsing, Maps preserving the nilpotency of products of operators, Linear Algebra Appl. 424 (2007) 222-239.

[16] L. N. Trefethen, M. Embree, Spectra and Pseudospectra: The Behavior of Nonnormal Matrices and Operators, Princeton University Press, Princeton, 2005. 